\title{
Nanoscale mapping of confinement potentials in single semiconductor quantum wires by near-field optical spectroscopy
}

\author{
Ch. Lienau, A. Richter, G. Behme, M. Süptitz, D. Heinrich, and T. Elsaesser \\ Max-Born-Institut für Nichtlineare Optik und Kurzzeitspektroskopie, D-12489 Berlin, Germany \\ M. Ramsteiner, R. Nötzel, and K. H. Ploog \\ Paul-Drude-Institut für Festkörperelektronik, D-10117 Berlin, Germany \\ (Received 24 December 1997; revised manuscript received 10 April 1998)
}

\begin{abstract}
The quasi-one-dimensional confinement potential and its influence on carrier transport in a GaAs quantum wire structure are directly mapped by low-temperature near-field scanning optical spectroscopy with subwavelength spatial resolution. Shallow asymmetric potential barriers in the vicinity of the quantum wire are detected, and their height and width are determined quantitatively. We demonstrate the strong influence of such local barriers on carrier transport and trapping into the quantum wire, suppressing carrier trapping at low temperature. [S0163-1829(98)05827-5]
\end{abstract}

The low-dimensional confinement of carriers in semiconductor nanostructures is based on spatial variations of the band structure, e.g., the band gap, on a nanometer scale by a controlled change of the material composition. To study such confinement potentials, experimental techniques with a spatial resolution in the nanometer range are required. Until now, methods based on the injection of electrons, such as ballistic electron emission microscopy, ${ }^{1,2}$ which was derived from scanning tunneling microscopy, ${ }^{3}$ or cathodoluminescence spectroscopy, ${ }^{4}$ have mainly been used for monitoring the local band structure of subsurface nanostructures. However, the initial energy distribution of carriers, its relaxation toward quasiequilibrium, and the transport of carriers in the nanostructure are difficult to control in such measurements. In contrast, all-optical spectroscopy allows for resonant excitation and-thus-for generation of carriers under welldefined conditions. To make use of these advantages, one has to overcome the diffraction limit of conventional optical microscopy, e.g., by using near-field optical techniques with a spatial resolution of about $100 \mathrm{~nm} .^{5,6}$ The tremendous potential of near-field spectroscopy, in particular at low temperatures, for the microscopic analysis of semiconductor nanostructures was first demonstrated in experiments on quantum wells $^{7}$ (QW's) and quantum wires ${ }^{8}$ (QWR's). Very recently, the absorption properties of single quantum wires have been investigated using near-field spectroscopy., 9

In this paper, we report a study combining lowtemperature near-field optical microscopy and photoluminescence spectroscopy, to map directly the local confinement potential of a quasi-one-dimensional (1D) GaAs structure, i.e., a QWR. Local energetic barriers separating the QWR and the embedding QW are mapped, and their effect on the trapping of carriers from quasi-two-dimensional (2D) QW into quasi-1D QWR states is demonstrated in experiments at variable temperatures.

The QWR structure was grown by molecular-beam epitaxy on patterned GaAs (311)A substrates at the sidewall of 15-20-nm high mesa stripes oriented along [01-1]. ${ }^{11}$ It consists of a nominally 6-nm-thick GaAs QW layer clad by 50nm-thick $\mathrm{Al}_{0.5} \mathrm{Ga}_{0.5} \mathrm{As}$ barriers. Formation of a sidewall
QWR arises from the preferential migration of $\mathrm{Ga}$ atoms within the QW layer from both the mesa top and mesa bottom toward the sidewall. Cross-sectional TEM images ${ }^{11}$ indicate that - as a result of this migration process - the QW thickness near the sidewall increases from 6 to $13 \mathrm{~nm}$. This change in QW thickness results in a $1 \mathrm{D}$ confinement over a lateral QWR width of $50 \mathrm{~nm}$.

Spatially resolved near-field spectroscopic experiments were performed at temperatures between 10 and $300 \mathrm{~K}$ with a newly designed vacuum near-field scanning optical microscope. ${ }^{12}$ In this instrument, subwavelength spatial resolution is achieved by transmitting the excitation light through an aperture with a diameter of about $150 \mathrm{~nm}$ at the end of a near-field fiber probe. Photoluminescence (PL) emitted by the sample is collected in the far field in reflection geometry through a conventional microscope objective. At the temperatures of this study, the QWR exciton diffusion length is significantly larger than the diameter of the metal-coated fiber probe. Thus a far-field collection of QWR luminescence is only weakly disturbed by the presence of the fiber probe. The luminescence is dispersed in a $0.25-\mathrm{m}$ double monochromator with a spectral resolution of $1.2 \mathrm{~nm}$ and detected with a silicon avalanche photodiode. Different tunable lasers served for excitation of the sample with powers between 10 and $100 \mathrm{nW}$, corresponding to very low carrier densities of $10^{4}-10^{5} \mathrm{~cm}^{-1}$.

In a first series of measurements, spatially resolved photoluminescence excitation (PLE) spectra were recorded in order to map the local energetics of the QWR and the embedding QW structure. At a sample temperature of $10 \mathrm{~K}$, ${ }^{10}$ the QWR emission appears as a single narrow peak centered at $1.545 \mathrm{eV}$ with a spectral full width at half maximum of 8 $\mathrm{meV}$. The PLE spectrum is spatially confined to the QWR region, and the PLE intensity exhibits several maxima below the onset of QW absorption at $1.63 \mathrm{eV}$. Such peaks are due to interband transitions between the quasi-one-dimensional valence and conduction subbands of the QWR. ${ }^{13}$ Calculations within the adiabatic approximation ${ }^{14}$ suggest that the first peaks are due to the $n=1(1.558 \mathrm{eV}), n=2(1.570 \mathrm{eV})$, and $n=3(1.583 \mathrm{eV})$ heavy-hole to conduction-band transi- 
tions. The splitting between the $n=1$ and 2 transition is on the order of $12 \mathrm{meV}$, as expected for a QWR width of about $50 \mathrm{~nm}$. These calculations indicate a confinement potential energy of $80 \mathrm{meV}$, in agreement with the experimental finding. The lateral $y$ dependence of the QWR confinement energies of electrons and holes can be approximated as $U_{i}(y)$ $=\varepsilon_{i}(0)+\left[\varepsilon_{i}(\infty)-\varepsilon_{i}(0)\right] \tanh ^{2}(a y)$, with a full width at half maximum of $2 \operatorname{arctanh}(0.5) / a$ of about $50 \mathrm{~nm}$. This shape of the potential is in good agreement with TEM images of this structure. ${ }^{11}$ These images indicate a thickness variation of the GaAs layer from 6 to $13 \mathrm{~nm}$ over a lateral distance of about $50 \mathrm{~nm}$ in the QWR region. The small Stokes shift of $13 \mathrm{meV}$ between the first peak and the PL maximum and the small width of the PL spectrum indicate the high structural quality of the sample. The inhomogeneous broadening of the spectra is limited by the structural properties of the corrugated (311) $A$ surface, showing typical corrugation lengths of about $32 \AA .{ }^{15}$ Thus emission is comprised of contributions from different localized excitons, as observed for QW's on low-index GaAs surfaces. ${ }^{16,7}$ Because of the short corrugation length, such components are not individually resolved on a 100 -nm length scale.

The spatially resolved PLE spectrum changes drastically as the sample temperature is increased to $77 \mathrm{~K}$. In Fig. 1(a), the luminescence intensity detected at $1.533 \mathrm{eV}$ near the maximum of the QWR emission is plotted as a function of excitation energy and position as the tip is scanned perpendicular to the QWR. The QWR PLE spectrum appears again as a spatially narrow peak around $y=0$, slightly redshifted with respect to the $10-\mathrm{K}$ spectrum. In contrast to the lowtemperature spectrum, strong QWR luminescence is now detected for QW excitation at energies higher than $1.6 \mathrm{eV}$ and at distances $y$ of several $\mu \mathrm{m}$ away from the QWR location. The spectral shape of the PLE spectrum for $y \neq 0$ changes strongly with excitation position. In particular, the lowest maximum of the PLE spectrum shifts toward higher photon energies as the excitation tip approaches the QWR location at $y=0$. In Fig. 1(b), we present cross sections through the image of Fig. 1(a) for different separations from the QWR on the mesa top $(y>0)$ (symbols). The peak in the spectra which is due to the excitonic enhancement of QW absorption shifts by about $14 \mathrm{meV}$ to higher energies as one moves from a distance of $y=5.5 \mu \mathrm{m}$ to the location of the QWR. On the mesa bottom $(y<0)$, this shift is even more pronounced, with PLE maxima at $1.610 \mathrm{eV}$ for $y=-3.5 \mu \mathrm{m}$ and 1.628 $\mathrm{eV}$ for $y=-0.4 \mu \mathrm{m}$.

The data of Fig. 1 demonstrate that carriers locally generated within the QW undergo real-space transfer to the location of the QWR, where they are trapped into quasi-onedimensional QWR states and contribute to the QWR emission. The time scale of real-space transfer is given by the recombination lifetime of carriers within the QW. Timeresolved PL measurements under the same excitation conditions give a lifetime of $1.2 \mathrm{~ns}$ at $77 \mathrm{~K}$. This time scale is much longer than typical thermalization and cooling times of carriers in the femtosecond and picosecond regime. As a result, the carriers undergoing real-space transfer form a quasiequilibrium distribution with a temperature close to lattice temperature. ${ }^{17}$

The PLE spectra recorded on the QW are closely related to the local QW absorption spectrum at the specific excita-

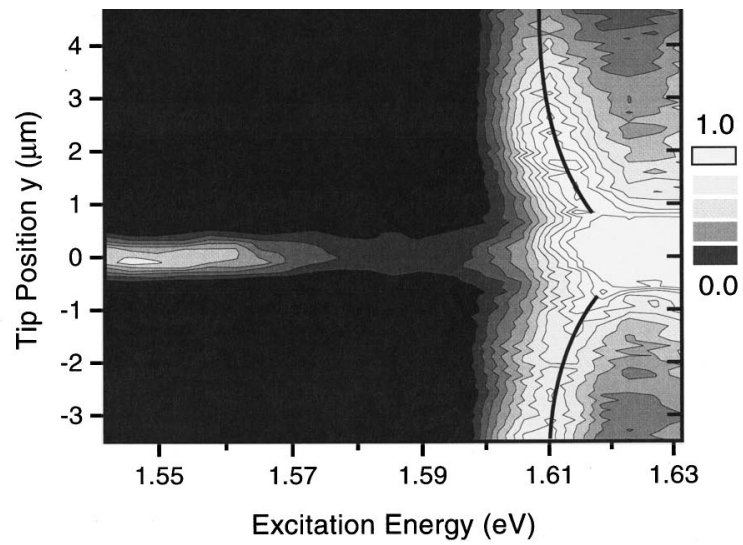

(b)



FIG. 1. (a) Near-field PLE spectrum at a sample temperature of $77 \mathrm{~K}$ and a detection energy of $1.533 \mathrm{~V}$, close to the maximum of the QWR emission at this temperature. Approaching the QWR at $y=0$, one observes a pronounced blueshift of the excitonic absorption peak of the quantum well (QW). (b) Cross sections through (a). Near-field mesa top QW PLE spectra at fixed excitation positions relative to the QWR location at $y=0$ (solid circles). The solid lines were calculated from Eq. (1).

tion position. The results in Fig. 1(b) reveal a pronounced excitonlike feature in the QW absorption, and a blueshift of this maximum with decreasing separation from the QWR. For a quantitative analysis, we used the Elliott formula ${ }^{18}$ for the absorption spectrum $\alpha(E)$ of a quasi-two-dimensional semiconductor:

$$
\begin{aligned}
\alpha(E) \propto & \frac{E}{E_{0}}\left[\sum_{n=0}^{\infty} \frac{4}{(n+1 / 2)^{3}} \delta\left(\Delta+\frac{1}{(n+1 / 2)^{3}}\right)\right. \\
& \left.+\Theta(\Delta) \frac{\exp (\pi / \sqrt{\Delta})}{\cosh (\pi / \sqrt{\Delta})}\right] .
\end{aligned}
$$

Here $E$ is the excitation energy, $n$ the quantum number of the bound exciton states, and $E_{0}$ the $2 \mathrm{D}$ exciton binding energy. $\Delta=\left(E-E_{g}\right) / E_{0}$, with $E_{g}$ being the band gap energy. $\Theta(\Delta)$ denotes the Heaviside step function. The dominant features are the $n=0$ exciton absorption peak and the continuum absorption, i.e., the second term in Eq. (1). At each excitation 




FIG. 2. Lateral band-gap profile as obtained from an analysis of the QW PLE spectrum shown in Fig. 3. The lateral shift in excitonic QW absorption peak corresponds to a decrease of the average QW thickness in the region close to the QWR. This thinning is a direct consequence of the $\mathrm{Ga}$ atom migration toward the sidewall during the growth process, and is most pronounced on the mesa bottom. Inset: QWR confinement potential as derived from the data in (a), and calculated energies of the 1D subbands (lines).

position, the spectra in Fig. 1(b) were analyzed including a Gaussian-shaped inhomogeneous broadening of the spectra due to the interface roughness of the QW. The solid lines in Fig. 1(b) represent the calculated spectra, which are in good agreement with the experimental results. The calculation gives the local band-gap energy $E_{g}(y)$ as a function of excitation position $y .{ }^{19} \mathrm{~A}$ plot of the band-gap energies of the QW versus $y$ is shown in Fig. 2. On each side of the wire, the potential shows two pronounced maxima, separated by approximately $1 \mu \mathrm{m}$. On the mesa bottom $(y<0)$ the band gap increases by $18 \mathrm{meV}$ over a length scale of about $2 \mu \mathrm{m}$ as it approaches the QWR, while on the mesa top $(y>0)$ the band-gap shift is slightly less pronounced and amounts to about $14 \mathrm{meV}$.

The change in band-gap energy originates mainly from a change in the average local thickness of the QW, that decreases from about $5.6 \mathrm{~nm}$ on the flat-area mesa top and bottom down to $4.8(y<0)$ and $5.0 \mathrm{~nm}(y>0)$ in the vicinity of the wire (Fig. 2). This thinning is a consequence of the $\mathrm{Ga}$ atom migration toward the sidewall during the growth process, and is determined by the specific molecular-beamepitaxy growth parameters, e.g., the substrate temperature. The asymmetry in the band-gap profile on the mesa top ( $y$ $>0)$ and bottom $(y<0)$ is most likely related to the wet chemical etching procedure that is used to pattern the mesa structure, ${ }^{11}$ and the resulting changes of the mobility of Ga atoms during growth.
Based on these results, we derive the complete confinement potential of the quantum-well-embedded QWR. Two different spatial regions are distinguished. In the region $|y|>100 \mathrm{~nm}$, the potential can directly be taken from Fig. 2, while in the central $100 \mathrm{~nm}$ around $y=0$ the width of the potential is taken from the PLE spectrum at $10 \mathrm{~K}$, and independently from the cross-sectional TEM images. ${ }^{11}$ In the inset of Fig. 2, the potential is plotted as a function of $y$ for the whole interval between -2 and $+2 \mu \mathrm{m}$. Using this information and a ratio of 2:1 for the conduction-to-valence-bandoffset energy in the $2 \mathrm{D} \mathrm{GaAs} / \mathrm{Al}_{0.5} \mathrm{Ga}_{0.5} \mathrm{As}$ structure, the quasi-one-dimensional subband structure and the interband transition energies of the QWR can now be calculated within the adiabatic approximation. The 1D subband energies, presented in the inset of Fig. 2 (lines), are in agreement with the low-temperature PLE spectrum. ${ }^{10} \mathrm{We}$ note that Coulomb correlation effects are neglected in these calculations.

The height of the local barriers of about $15 \mathrm{meV}$ is only a small fraction of the QWR confinement energy of $80 \mathrm{meV}$, and the barrier maxima are separated by $1 \mu \mathrm{m}$. As a result, the influence of the barriers on the energetic position of the lowest QWR subbands is rather small. Nonetheless, the presence of the barriers strongly influences the carrier transport within the sample, as is evident from the photoluminescence measurements discussed next. Near-field PL spectra were recorded at various sample temperatures with spatially re- 

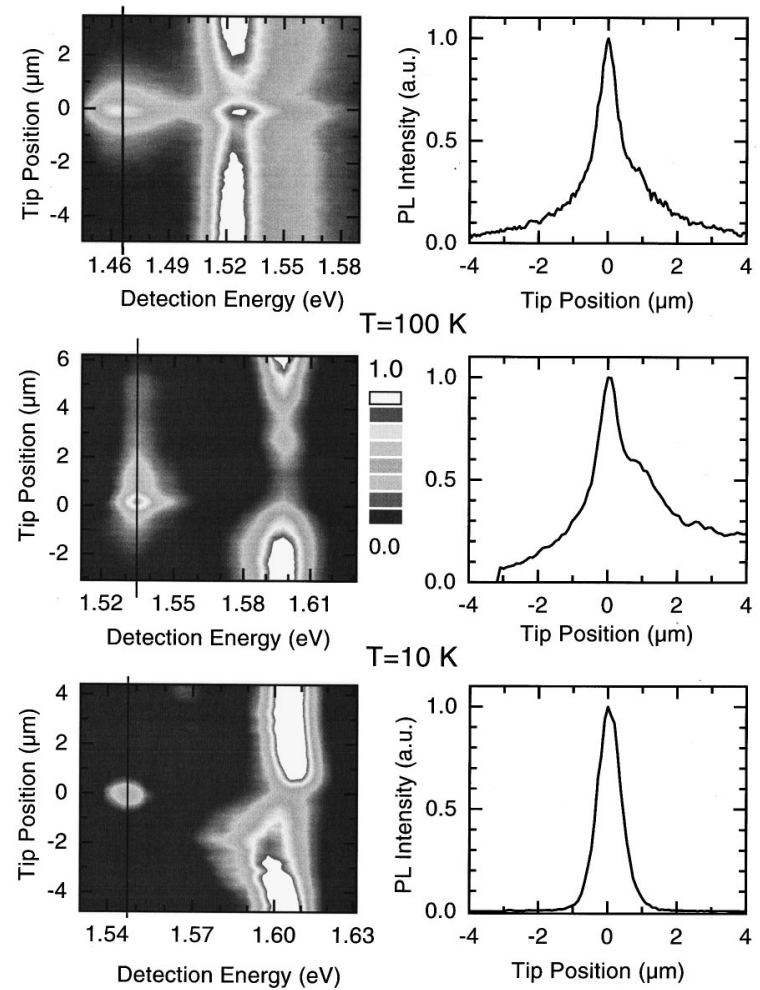

FIG. 3. (a) Near-field photoluminescence spectra of the QWR sample for localized excitation at $1.595 \mathrm{eV}$ at three different sample temperatures of 300,100 , and $10 \mathrm{~K}$. The luminescence intensity is plotted as a function of detection energy (abscissa) and of lateral distance between the QWR located at $y=0$ and the fiber tip (ordinate). (b) Spatial cross sections through the near-field PL spectra at the energy of the QWR emission maximum.

solved excitation at a photon energy of $1.959 \mathrm{eV}$ (Fig. 3). At $300 \mathrm{~K}$ [Fig. 3(a)], QWR emission appears as a peak around $1.47 \mathrm{eV}$. The cross section through the QWR PL spectrum shows (i) a narrow peak at $y=0$ arising from direct QWR excitation, and (ii) broad tails that decay exponentially over a length of several micrometers in the QW area. This occurrence of QWR luminescence after localized QW excitation involves ambipolar carrier drift diffusion within the QW, and subsequent trapping into the QWR, as was discussed in Ref. 10. The local current density that determines the carrier transport is the sum of a diffusion current, arising from the concentration gradient $\nabla n$ within the sample $j_{\text {diff }}=e D \nabla n$ and a drift current $j_{\text {drift }}=n e \mu_{\text {eff }} E$ due to the local internal electric field $E$ (of up to $200 \mathrm{~V} / \mathrm{cm}$ ) that originates from the presence of the energetic barriers. Using the Einstein relation $j_{\text {diff }}=\mu_{\text {eff }} k T \nabla n$ (e is the electron charge, $k T$ the thermal energy). At $300 \mathrm{~K}$, the influence of the drift current is weak, and the carrier transport is mainly of diffusive character. An ambipolar diffusion coefficient of $D=12.2 \mathrm{~cm}^{2} / \mathrm{s}$ and an effective mobility $\mu_{\text {eff }}=488 \mathrm{~cm}^{2} / \mathrm{V}$ s are derived from the data. This $\mu_{\text {eff }}$ is close to the hole mobility in GaAs at $300 \mathrm{~K}$, and thus hole diffusion determines the real-space transfer. At low temperatures, a drastically different behavior is found. At 10 $\mathrm{K}$ [Fig. 3(c)], QWR emission (at $1.545 \mathrm{eV}$ ) appears as a single narrow peak centered around $y=0$ that is well represented by a Gaussian profile of a width of about $850 \mathrm{~nm}$. At $10 \mathrm{~K}$, the drift current in the regions of the barriers, that is, opposing the diffusive motion of carriers from the point of their generation towards the QWR, is larger than the diffusive current. Thus the real-space transfer of carriers across the barriers is strongly suppressed, and trapping of carriers generated within the flat area $\mathrm{QW}$ is prohibited. At intermediate temperatures around $100 \mathrm{~K}$ [Fig. 3(b)], the spatial dependence of the QWR luminescence peak around $1.54 \mathrm{eV}$ becomes strongly asymmetric, directly reflecting the effect of the spatial asymmetry of the band-gap potential on the carrier transport. On the mesa bottom $(y<0$, barrier height $18 \mathrm{meV}$ ), the QWR luminescence is significantly less intense than on the mesa top $(y>0$, barrier height $14 \mathrm{meV})$. At this temperature, both drift and diffusive currents are of similar magnitude, and the fraction of the carriers that can pass the barrier and relax into the QWR thus depends strongly on the strength of the local internal electric field, and thus is markedly different on both sides of the QWR. Because of the high mobility of holes of about $7000 \mathrm{~cm}^{2} / \mathrm{V}$ s at $100 \mathrm{~K}$ and the long 2D carrier lifetime of $1.4 \mathrm{~ns}$, the diffusion length over which carriers are collected is about $3 \mu \mathrm{m}$.

In conclusion, we have demonstrated the potential of lowtemperature near-field photoluminescence excitation spectroscopy for mapping the local band profile in semiconductor nanostructures with subwavelength spatial resolution. The confinement potential for a quantum-well embedded GaAs quantum wire structure grown on patterned (311)A GaAs surfaces was determined by this all-optical technique. Shallow asymmetric potential barriers were identified, and the influence of the potential on carrier transport and trapping was directly demonstrated. Similar barriers are likely to play an important role in the carrier transport and trapping in quantum wires grown by other techniques, in particular in those on V-shaped GaAs substrates. ${ }^{20}$ Further improvement of near-field optical microscopy, e.g., toward higher spatial resolution, and the combination with time-resolved spectroscopy, will make this powerful technique a versatile tool for studying the local electronic properties and carrier dynamics in semiconductor nanostructures.
${ }^{1}$ W. J. Kaiser and L. D. Bell, Phys. Rev. Lett. 60, 1406 (1988).

${ }^{2}$ M. E. Rubin, G. Medeiros-Ribeiro, J. J. O’Shea, M. A. Chin, E. Y. Lee, P. M. Petroff, and V. Narayanamurti, Phys. Rev. Lett. 77, 5268 (1996).

${ }^{3}$ G. Binnig, H. Rohrer, Ch. Gerber, and E. Weibel, Phys. Rev. Lett. 49, 57 (1982).
${ }^{4}$ J. Christen, M. Grundmann, and D. Bimberg, J. Vac. Sci. Technol. B 9, 2358 (1991).

${ }^{5}$ D. W. Pohl, W. Denk, and M. Lanz, Appl. Phys. Lett. 44, 651 (1984).

${ }^{6}$ E. Betzig and J. K. Trautmann, Science 257, 189 (1992).

${ }^{7}$ H. F. Hess, E. Betzig, T. D. Harris, L. N. Pfeiffer, and K. W. 
West, Science 264, 1740 (1994).

${ }^{8}$ R. D. Grober, T. D. Harris, J. K. Trautman, E. Betzig, W. Wegscheider, L. Pfeiffer, and K. West, Appl. Phys. Lett. 64, 1421 (1994).

${ }^{9}$ T. D. Harris, D. Gershoni, R. D. Grober, L. Pfeiffer, K. W. West, and N. Chand, Appl. Phys. Lett. 68, 988 (1996).

${ }^{10}$ A. Richter, G. Behme, M. Süptitz, C. Lienau, T. Elsaesser, R. Nötzel, M. Ramsteiner, and K.-H. Ploog, Phys. Rev. Lett. 79, 2145 (1997).

${ }^{11}$ R. Nötzel, M. Ramsteiner, J. Menniger, A. Trampert, H.-P. Schönherr, L. Däweritz, and K. H. Ploog, Jpn. J. Appl. Phys., Part 2 35, L297 (1996).

${ }^{12}$ G. Behme, A. Richter, M. Süptitz, and Ch. Lienau, Rev. Sci. Instrum. 68, 3458 (1997).

${ }^{13}$ F. Rossi and E. Molinari, Phys. Rev. Lett. 76, 3642 (1996).

${ }^{14}$ E. Kapon, D. M. Hwang, and R. Bhat, Phys. Rev. Lett. 63,
430 (1989).

${ }^{15}$ R. Nötzel, N. N. Ledentsov, L. Däweritz, K. Ploog, and M. Hohenstein, Phys. Rev. B 45, 3507 (1992).

${ }^{16}$ D. Gammon, E. S. Snow, B. V. Shanabrook, D. S. Katzer, and D. Park, Science 273, 87 (1996).

${ }^{17}$ A. Richter, M. Süptitz, Ch. Lienau, and T. Elsaesser (unpublished).

${ }^{18} \mathrm{H}$. Haug and S. W. Koch, Quantum Theory of the Optical and Electronic Properties of Semiconductors (World Scientific, Singapore, 1997).

${ }^{19}$ The change in quantum-well thickness near the QWR is correlated with an increase in inhomogeneous broadening. This is directly observed as a pronounced increase in the spectral width of the $n=1$ exciton absorption peak.

${ }^{20}$ F. Vouilloz, D. Y. Oberli, M.-A. Dupertuis, A. Gustafsson, F. Reinhardt, and E. Kapon, Phys. Rev. Lett. 78, 1580 (1997). 